\title{
Case Report: A Case of Tuberculosis with Psoriasiform Lichen Scrofulosorum Exhibiting Koebner's Phenomenon followed by Scrofuloderma
}

\author{
Ishika Muradia, ${ }^{1}$ Geeti Khullar, ${ }^{1 *}$ Shruti Sharma, ${ }^{2}$ and Sachin Kolte ${ }^{3}$ \\ ${ }^{1}$ Department of Dermatology and Sexually Transmitted Diseases, Vardhman Mahavir Medical College and Safdarjung Hospital, Delhi, India; \\ ${ }^{2}$ National Institute of Pathology (Indian Council of Medical Research), Safdarjung Hospital Campus, Delhi, India; ${ }^{3}$ Department of Pathology, \\ Vardhman Mahavir Medical College and Safdarjung Hospital, Delhi, India
}

\begin{abstract}
Lichen scrofulosorum is the most common tuberculid in the Indian population and the second most common form of cutaneous tuberculosis. We discuss an uncommon presentation of lichen scrofulosorum, with psoriasiform morphology and koebnerization at tuberculin test site, associated with cervical lymphadenitis in a 17-year-old girl. Although the cutaneous lesions resolved completely after 3 months of antitubercular treatment (ATT), she developed scrofuloderma in the left cervical region at 4 months. In the absence of rifampicin resistance, ATT was continued for another 6 months, with no further evidence of disease activity. This case represents an infrequent occurrence of lichen scrofulosorum followed by scrofuloderma, which necessitated a prolonged course of first-line ATT.
\end{abstract}

\section{INTRODUCTION}

Lichen scrofulosorum is a micropapular tuberculid resulting from a type IV hypersensitivity reaction to hematogenous dissemination of Mycobacterium tuberculosis and occasionally other non-tubercular mycobacteria. Varied morphological presentations of lichen scrofulosorum are described. ${ }^{1-4}$ Koebner's phenomenon, characterized by the development of isomorphic lesions at sites not affected by the disease following trauma, is exceedingly rare in lichen scrofulosorum. ${ }^{5}$ An internal focus of tuberculosis, particularly a lymph node, can be identified in a large majority of individuals with lichen scrofulosorum. Scrofuloderma occurs as a result of cutaneous involvement from an underlying contiguous focus of tuberculosis, most frequently a lymph node. Lichen scrofulosorum may be associated with other forms of cutaneous tuberculosis, often those with high immunity such as lupus vulgaris and tuberculosis verrucosa cutis, and less commonly with scrofuloderma.

\section{CASE REPORT}

A 17-year-old girl presented with slightly itchy, erythematous pinhead-size papules on the trunk for 1 month. There was no history of cough, fever, night sweats, weight loss, or any other systemic complaints. She denied any past or family history of tuberculosis. Physical examination revealed multiple discrete to grouped erythematous perifollicular papules with minimal white scaling (Supplemental Figure 1), that were coalescing to form ill-defined plaques on the chest, abdomen (Figure 1), and lower back. A single discrete, firm, non-tender lymph node measuring $2 \times 2 \mathrm{~cm}$ was palpable in the left cervical region. A Bacille Calmette-Guerin scar was present on her left arm. A Mantoux test was strongly positive, with an induration of $24 \times 27 \mathrm{~mm}$, along with psoriasiform plaque around the margins of the positive reaction on the flexor aspect of her left forearm (Figure 2). Systemic examination was unremarkable. Histopathological examination from a papule on the abdomen showed a non-caseating epithelioid cell granuloma replacing the hair follicle with a remnant of

*Address correspondence to Geeti Khullar, Department of Dermatology and Sexually Transmitted Diseases, Vardhman Mahavir Medical College and Safdarjung Hospital, Delhi, India. E-mail: geetikhullar@yahoo.com arrector pili muscle in the vicinity (Figure 3 and Supplemental Figure 2). Special stain, tissue culture, and polymerase chain reaction were all negative for $M$. tuberculosis. Fine-needle aspiration cytology from a left cervical lymph node revealed granulomatous lymphadenitis (Supplemental Figure 3). No acid-fast bacilli were seen on Ziehl-Neelsen stain. Routine investigations, including hemogram, and liver and renal function tests, were within normal limits, except for a mildly increased erythrocyte sedimentation rate of $25 \mathrm{~mm} / \mathrm{h}$. Serologies for HIV, and hepatitis B and C viruses were negative. Chest X-ray and abdominal ultrasound did not reveal any abnormalities. Based on clinical presentation, histological findings, and other investigations, a final diagnosis of psoriasiform lichen scrofulosorum with left cervical tubercular lymphadenitis was established. The patient was started on antitubercular treatment (ATT) first-line agents in a fixeddose combination per kilogram body weight to be taken for 6 months. For the first 2 months (intensive phase), she received $225 \mathrm{mg}$ isoniazid, $450 \mathrm{mg}$ rifampicin, $1,200 \mathrm{mg}$ pyrazinamide, and $825 \mathrm{mg}$ ethambutol daily; and isoniazid, rifampicin, and ethambutol for the next month (continuation phase). At 3 months, although complete resolution of cutaneous lesions occurred (Supplemental Figures 4 and 5), cervical lymphadenitis did not show any improvement. While continuing ATT, at 4 months the patient developed a single, illdefined, painful erythematous swelling (size, $6 \times 4 \mathrm{~cm}$ ), with pus-discharging sinuses on the left side of the neck (Figure 4). Skin biopsy revealed acanthotic epidermis. Dermis showed dilated capillaries and dense inflammatory infiltrate comprised of epithelioid cell granuloma with lymphocytes and Langhans giant cells (Supplemental Figures 6 and 7). Tissue culture on a mycobacteria growth indicator tube showed growth of $M$. tuberculosis. A cartridge-based nucleic acid amplification test performed on the aspirated pus was positive for $M$. tuberculosis; rifampicin resistance was not detected. A diagnosis of cervical scrofuloderma was made. The patient was continued on the three-drug ATT for an additional 6 months. After a total of 10 months of treatment, the lesion healed with puckered scarring (Supplemental Figure 8).

\section{DISCUSSION}

Lichen scrofulosorum classically presents as asymptomatic, grouped perifollicular skin-colored to erythematous 


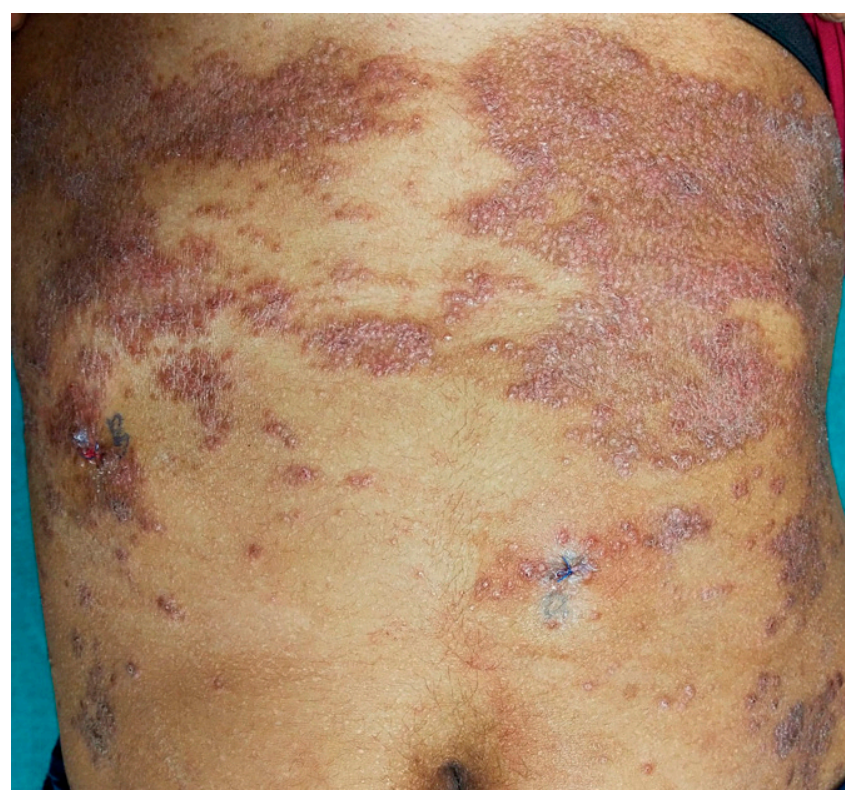

FIGURE 1. III-defined erythematous to brownish plaques with fine adherent scales and few discrete papules in the periphery. This figure appears in color at www.ajtmh.org.

papules on the trunk and proximal extremities in children and adolescents. Minimal scaling, crusting, and micropustules may be present. ${ }^{1,2}$ Atypical morphologies, such as psoriasiform, lichenoid, granuloma annulare-like and noduloulcerative, have been reported occasionally. ${ }^{1-4}$ Severe inflammatory scaly plaques with few pustules have been associated with disseminated tuberculosis. ${ }^{2}$ In our patient, the lesions were perifollicular papules to start with, then manifested

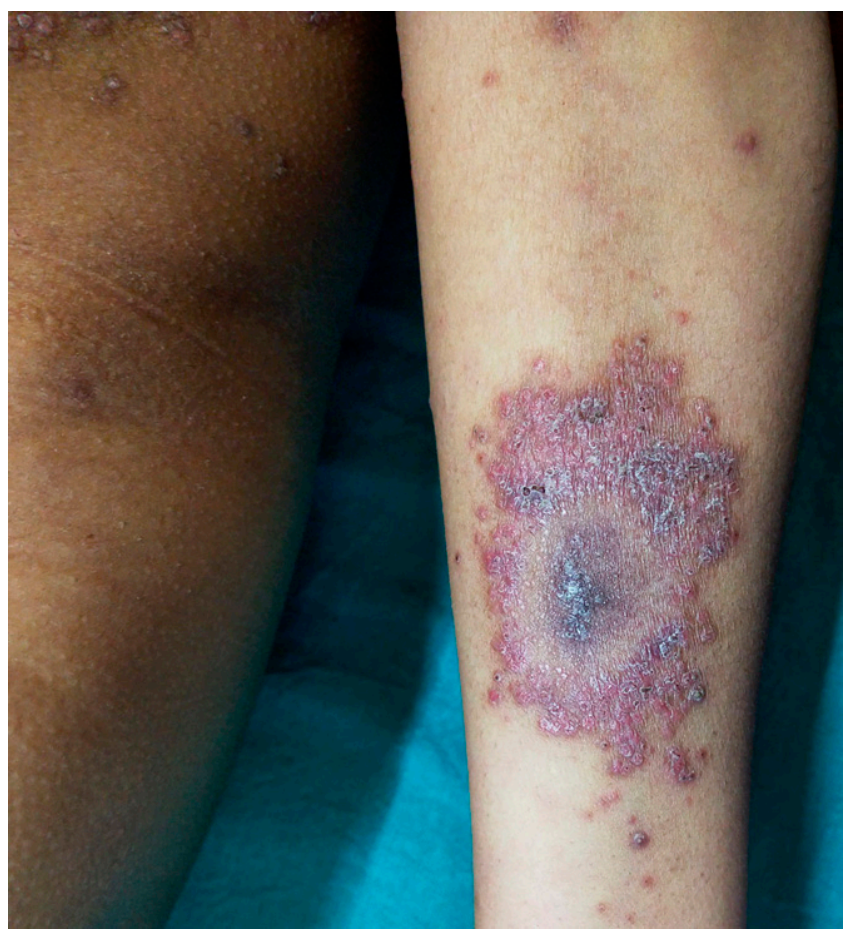

FIGURE 2. Strongly positive Mantoux reaction with koebnerization of lichen scrofulosorum as scaly plaque and papules in the periphery of a positive reaction. This figure appears in color at www.ajtmh.org.

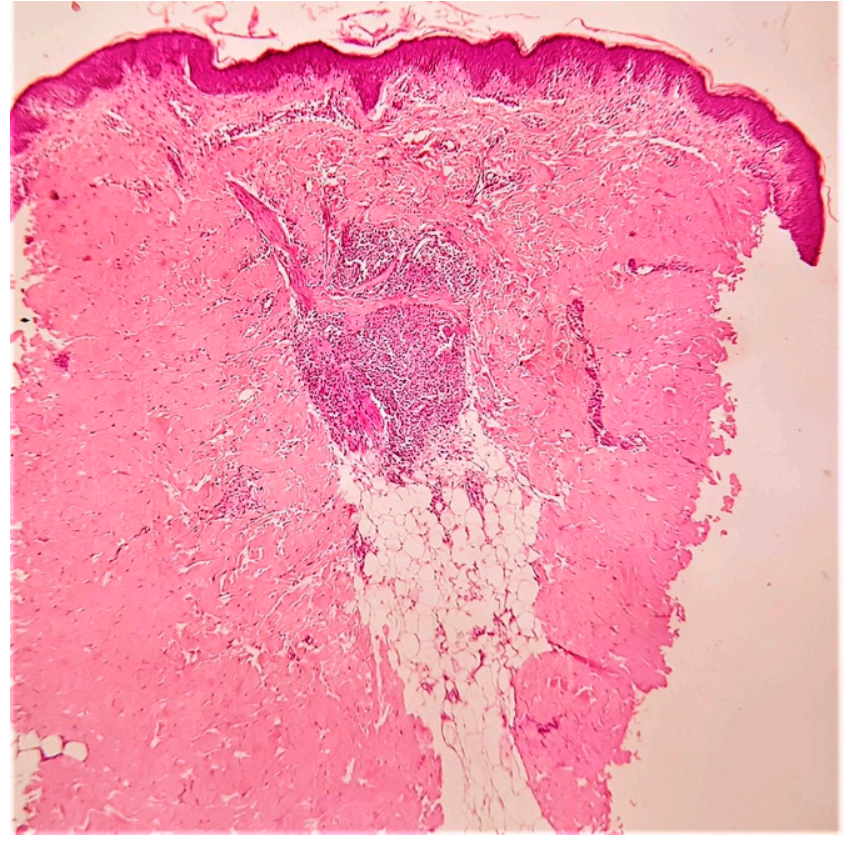

FIGURE 3. Epidermis is unremarkable. Dermis shows perivascular lymphohistiocytic infiltrate in the papillary dermis and epithelioid cell granuloma around the arrector pili muscle in the mid dermis (hematoxylin-eosin stain, $\times 40$ magnification). This figure appears in color at www.ajtmh.org.

subsequently as large erythematous plaques with grayish white adherent scales, resembling psoriasis.

Koebner's phenomenon is known to occur in many dermatological conditions; however, it is not commonly reported in

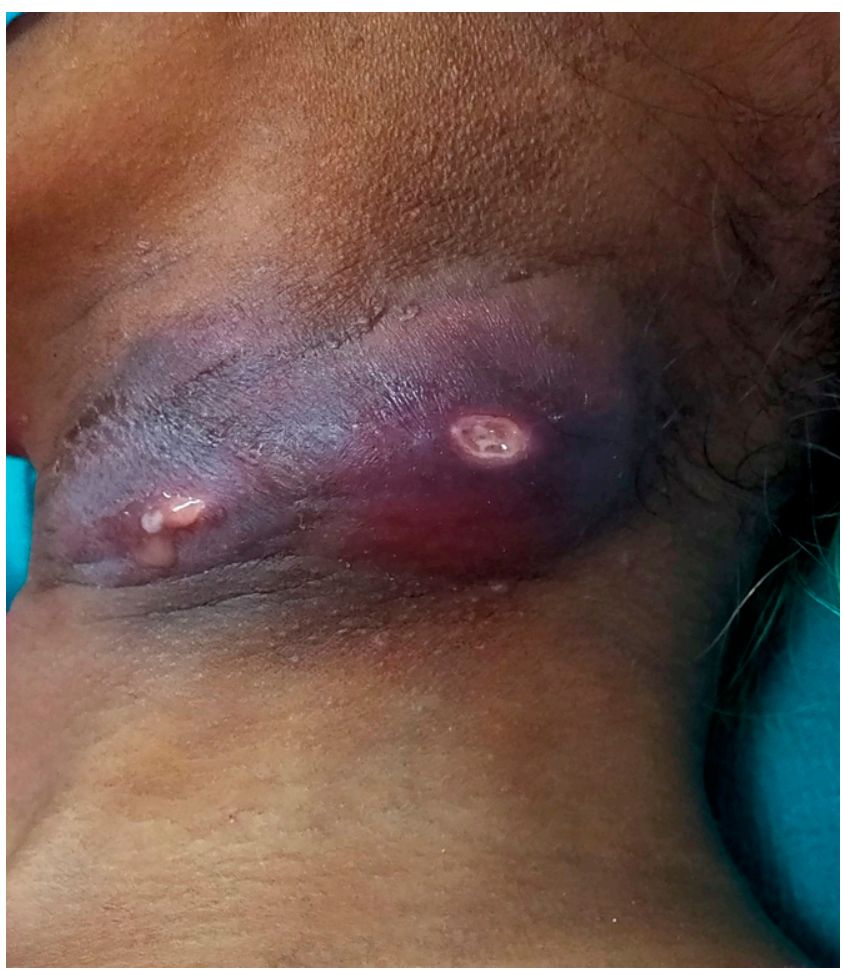

FIGURE 4. Erythematous swelling of size $6 \times 4 \mathrm{~cm}$, with pusdischarging sinuses over left side of neck. This figure appears in color at www.ajtmh.org 
lichen scrofulosorum. ${ }^{5}$ Epidermal injury at the tuberculin skin test site has been shown to induce Koebner's phenomenon in psoriasis, autoimmune blistering diseases, and, rarely, in tuberculids. ${ }^{5-8}$ Our patient had strongly positive tuberculin reaction at 48 hours, along with koebnerization of lesions of lichen scrofulosorum around that site, both resulting from a type IV delayed hypersensitivity reaction to a mycobacterial antigen. The release of tumor necrosis factor- $\alpha$ and interferon- $\alpha$ induced by a tuberculin reaction have also been implicated pathogenetically in causing lichen scrofulosorum. ${ }^{8}$ Furthermore, mycobacterial heat shock protein stimulates the immune response, resulting in lesions of lichen scrofulosorum. ${ }^{8}$

An underlying focus of tuberculosis can be detected in $72 \%$ of the cases of lichen scrofulosorum, with lymph nodes being the most common site, followed by bones and lungs. ${ }^{9}$ Our patient had granulomatous lymphadenitis of the left cervical region. Lichen scrofulosorum has been described infrequently as coexisting with scrofuloderma. ${ }^{4,9,10}$ In a series of 39 patients, only four patients (10\%) had concomitant scrofuloderma. ${ }^{9}$ However, development of scrofuloderma while on ATT and after lichen scrofulosorum had resolved is an unusual finding in our patient. Rifampicin resistance was not detected and it is likely that cervical lymphadenitis was slow to respond initially to ATT, possibly because of a high mycobacterial load, and thereby manifested with a contiguous spread to the overlying skin in due course. Treatment for a prolonged duration during the continuation phase healed the scrofuloderma and underlying lymphadenitis completely. Tuberculous lymphadenitis may respond slowly to treatment, and the nodes can enlarge during or even after stopping treatment. ${ }^{11}$ A subgroup of cutaneous tuberculosis patients categorized as slow responders has been observed to demonstrate clinical improvement after 60 to 123 days of therapy. ${ }^{12}$

In conclusion, the unique features in our patient were a psoriasiform subtype of lichen scrofulosorum, Koebner's phenomenon at the Mantoux site, the onset of scrofuloderma while on ATT, and a favorable response to prolonged therapy.

Received March 1, 2021. Accepted for publication May 4, 2021.

Published online July 27, 2021.

Note: Supplemental figures appear at www.ajtmh.org.

Acknowledgment: The American Society of Tropical Medicine and Hygiene (ASTMH) assisted with publication expenses.
Authors' addresses: Ishika Muradia and Geeti Khullar, Department of Dermatology and Sexually Transmitted Diseases, Vardhman Mahavir Medical College and Safdarjung Hospital, Delhi, India, E-mails: ishikathedermatologist.13@gmail.com and geetikhullar@yahoo.com. Shruti Sharma, National Institute of Pathology Indian Council of Medical Research, Safdarjung Hospital Campus, Delhi, India, E-mail: shrutigautam@rediffmail.com. Sachin Kolte, Department of Pathology, Vardhman Mahavir Medical College and Safdarjung Hospital, Delhi, India, E-mail: drsachinkolte@gmail.com.

\section{REFERENCES}

1. Dandale A, Gupta N, Dhurat R, Ghate S, 2013. Unusual presentation of lichen scrofulosorum. Indian J Dermatol Venereol Leprol 79: 436-438.

2. Kumar U, Sethuraman G, Verma P, Das P, Sharma VK, 2011. Psoriasiform type of lichen scrofulosorum: clue to disseminated tuberculosis. Pediatr Dermatol 28: 532-534.

3. Kondo M, Iwata Y, Numata S, Saito K, Watanabe S, Kobayashi T, Nagai A, Iwata T, Arima M, Sugiura K, 2018. Case of lichenoidlichen scrofulosorum: a rare variety of lichen scrofulosorum mimicking lichen planus in an elderly patient. $J$ Dermatol 45: e148-e149.

4. Otto Al, Harsing J, Herjavecz I, Kiss M, Karpati S, 2009. Scrofuloderma associated with granuloma annulare-like lichen scrofulosorum. Acta Dermatol Venereol 89: 640-642.

5. Pandhi D, Singal A, Wadhwa N, 2016. Lichen scrofulosorum: yet another disease manifesting the Koebner phenomenon? Int $J$ Dermatol 55: 809-810.

6. Bishnoi A, De D, Handa S, Mahajan R, Chatterjee D, Saikia UN, 2018. Bulla formation at the tuberculin skin test site in a patient with bullous pemphigoid: koebnerization or severe delayedtype hypersensitivity? Indian J Dermatol Venereol Leprol 84: 227-229.

7. Xu H, Li YM, Ma H, Gu WT, Chen ZQ, 2017. Mycobacterium tuberculosis found at both skin lesions and Mantoux testing site in a patient with erythema induratum of Bazin. J Dermatol 44: 11451147.

8. Chhabra G, Verma P, Sharma S, 2020. Koebner phenomenon induced by Mantoux testing in a case of papulonecrotic tuberculid. Trop Doct 50: 74-77.

9. Singal A, Bhattacharya SN, 2005. Lichen scrofulosorum: a prospective study of 39 patients. Int J Dermatol 44: 489-493.

10. Camacho D, Pielasinski U, Revelles JM, Gorgolas M, Manzarbeitia $F$, Kutzner H, Requena L, 2011. Lichen scrofulosorum mimicking lichen planus. Am J Dermatopathol 33: 186-191.

11. Gandhare A, Mahashur A, 2017. Tuberculosis of the lymph nodes: many facets, many hues. Astrocyte 4: 80-86.

12. Ramam M, Tejasvi T, Manchanda Y, Sharma S, Mittal R, 2007. What is the appropriate duration of a therapeutic trial in cutaneous tuberculosis? Further observations. Indian J Dermatol Venereol Leprol 73: 243-246. 